\title{
Development of an evacuation policy at a healthcare facility
}

\author{
Connor Barry BS, MBA, Zain Ali BS, MBA
}

\begin{abstract}
An Evacuation Policy is a procedure and plan that is needed for a healthcare facility to ensure the safety of patients, families, and the staff in the event of an Emergent or NonEmergent Evacuation. While disasters are unplanned, a procedure of any caliber can be used to maximize resources and abilities of a healthcare facility. An Evacuation Policy is the designated policy and procedure for a partial or complete evacuation of a hospital or clinic. During an evacuation, the organization and coordination of the evacuation policy are at top priority for the successful and safe relocation of patients, families, and staff. Without an effective policy, there would be an increased risk of chaos due to uncoordinated staff and patient movement. This paper proposes an efficient evacuation plan to ensure patients are evacuated in a smooth manner on and between floors. This proposed plan is based on four fundamental principles: staff hierarchy, universal patient triaging, patient movement procedure, and end point locations. Using these four principles, an efficient flow will be created for moving patients between the affected area and the safe end point. Evacuation plans are necessary for any emergency when mass coordinated patient and staff movement to safety is required.
\end{abstract}

Keywords: Disaster, hospital, evacuation, policy

\section{INTRODUCTION}

Development of an Evacuation Policy is a necessary and vital policy in the case of an evacuation of a hospital. The plan must be broad enough to cover all potential evacuation threats, but also specific enough to handle anything that might arise during the evacuation. However, prior to any evacuation, the healthcare facility needs to determine what the major threats are to the facility and develop an evacuation plan accordingly. For example, in Houston, the largest threats are hurricanes which produces high winds, flooding, and prolonged times without power. While it is difficult to plan for the exact event, the facility should be able to

\section{Corresponding author: Connor Barry}

Contact Information: Connor.barry@ttuhsc.edu

DOI: 10.12746/swrccc.v6i26.507 determine the greatest threats to its environment. The four main components of the Evacuation Policy are the staff hierarchy with specific job responsibilities, a universal triage system, patient movement procedure, and end point locations. By establishing these processes, a healthcare facility will be able to quickly and safely transport patients away from the affected facility.

\section{PROCESS}

\section{STAFF HIERARCHY AND RESPONSIBILITIES}

The largest component is the hierarchy because without a structure in place for the hospital staff, there will be a lack of coordinated movement of patients or staff. Figure 1 showcases the hierarchy that is encouraged by DQE (Indianapolis, Indiana), a corporation that specializes in disaster events for healthcare facilities. 


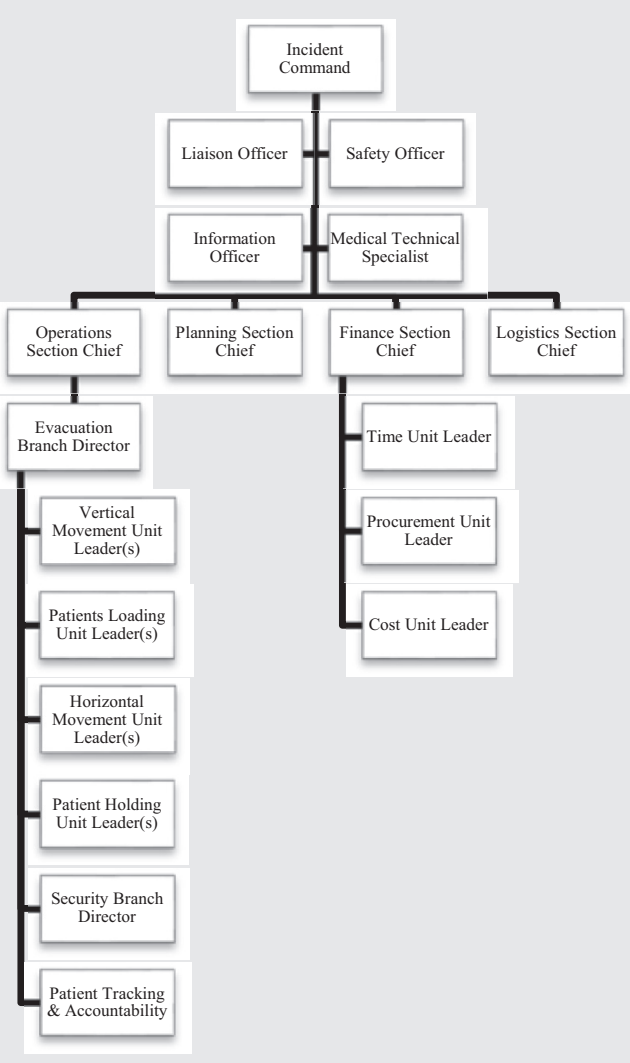

Figure 1. Hierarchy.

As seen in the figure, there are five key branches: Incident Command, Operations, Planning, Finance, and Logistics. Job responsibilities for each person need to be established for three time periods: Prior to Evacuation, During Evacuation, and Returning from Evacuation.

Incident Command is composed of the Liaison, Safety, Information, and Medical Technical Officers. This group is the directing body for all patient movements and personnel. Four Section Chiefs oversee four distinct responsibilities: Operations, Planning, Finance, and Logistics.

Operations is in charge of all patient movements and is led by the Operations Section Chief and Evacuation Branch Director. Actual movement of the patient is performed by two separate teams depending if it is a horizontal movement across a fire door or between floors. These movements are done by the respective Vertical and Horizontal Movement Teams and aided by the Patient Loading Units. Between patient movement the responsibilities for the patient are transferred to the Patient Holding Units. These Units are in charge of patient care until they are moved from their Holding Units to the End Point Location. During all of the patient movements, there is associated tracking of the patient by the Patient Tracking \& Accountability Team.

The Planning Chief is responsible for the planning of the evacuations both before and during the evacuation. They are in charge of doing the required training and teaching of the subject matter. The Finance Chief is responsible for the tracking of staff hours, expenses, and facility requirements. The Logistics Chief is responsible for conducting all additional required tasks that arise during the evacuation, such as contacting businesses to aid the evacuation.

\section{TRIAGE}

Before an evacuation all facilities will be responsible for selecting their own universal triage systems. Therefore, during the evacuation, hospital personnel must triage all patients to prioritize the movement of patients. There are many different triage systems, such as TRAIN, Triage by Resource Allocation for IN-patients. This specific triage system is based on pediatric patients but is easily adapted to adults based on TRAIN's equipment guidelines. TRAIN has four levels of triage dictated by the colors in increasing severity of blue, green, yellow, and red.

Blue is termed Stable. These patients have no need for monitoring, are on room air and all oral medications, and need no specialized equipment to transport them. These patients could also be termed ambulatory and will be able to be transported out of the affected facility by personal vehicle, bus, or van.

Green is termed Minimal Life Support. These patients are anyone with one or more of the following 
conditions: wheelchair bound, low flow oxygen cannula, feeding tube, and/or intermittent IV medications. These patients need minimal care under the supervisor of medical professionals. They must also be transported in a medical vehicle or bus from the affected site.

Yellow is termed Critical Care. These patients are on moderate levels of life support but they have stable vitals. They have the following conditions: chest tube, conventional ventilator, and/or external pacemaker. These patients have up to one IV pharmacy drip. These patients are likely from an ICU, are currently stable, and have the ability to stay awake. They must be transported offsite by trained medical professionals in an ambulance.

Red is termed Specialized. These patients are on a high level of life support and are unstable. They have specialized equipment that is not designed for transportation. The patients are also on multiple IV medication drips and external equipment. They must be transported by ambulance or military equipment with a likely team of specialists, such as the patient's physician, respiratory therapist, and paramedics.

The process of triaging a patient will be done by the patient's nurse(s) on a daily basis. This quick process will allow the department to be prepared before the disaster and allow personnel to allocate their resources in the moment of a disaster.

\section{Patient movement PROCEDURES}

The movement of the patient is dictated and performed by the Operations Section Group. The following Patient Movement Procedure is an example of one successfully approved by a large private hospital in Texas. The following 13 steps are the appropriate course of action for relocating a patient to a safe environment.

1. Hospital Command Center and Incident Command is activated for a total or partial evacuation of the affected facility.
2. Staff and patients are notified of the evacuation by overhead announcement and paging. The announcement will use plain language to clearly and appropriately describe the situation. All areas will assist in the evacuation as needed. Additional staff will report to the Labor Pool for assignment.

3. Charge Nurses or Supervisors of all areas will fax or send a runner with the forms dictating their Patient Census and Staff Availability.

4. The Labor Pool will be formed from the additional staff who have reported and determined through Staff Availability Forms.

5. From the formed Labor Pool, the following teams will be formed:
a. Horizontal Movement Unit
b. Vertical Movement Unit
c. Patient Holding Unit
d. Patient Loading Unit
e. Patient Tracking \& Accountability Unit

6. Triage the patient and disaster situation

7. The floor staff and Patient Loading Unit will make the patient's Document Pouch, prepare medications for the next 72 hours, and prepare the patient for the transport teams.

8. The Holding Area Unit will prepare four separate locations for the four different levels of triaged patients on each floor. These will be labeled:
a. Blue
b. Green
c. Yellow
d. Red

9. There will be teams designated to prepare the End Point Locations for each of the four triage levels. These will be located on the first floor in a similar manner discussed below.

10. The Horizontal Movement Team will move the patient from his/her room to the Holding Area specific to that level of triage. The horizontal movement team will wait with the patient until the care is taken over by the Holding Area team. 
Table 1.

\begin{tabular}{|l|l|l|}
\hline $\begin{array}{l}\text { Triage } \\
\text { Color }\end{array}$ & Example Location & Exit \\
\hline Blue & Main Lobby & Main Lobby Door \\
\hline Green & Axillary Lobby & Axillary Lobby Exit \\
\hline Yellow & Emergency Department & Side Exit \\
\hline Red & Emergency Department & Side Exit \\
\hline
\end{tabular}

a. The Horizontal Movement Team will place a sticker on the door to signify that the patient's room is clear and evacuated.

11. Presuming a floor change needs to be made, the Vertical Movement Team will pick up the patient from the Holding Area and transport him/her down to the End Point Location designated for the triage level.

12. The Vertical Movement Team will wait for the End Point Location staff to take over care for the patient. Once the End Point Location Staff has taken over, the patient will stay there until transport out of the facility or to the next safe location is determined.

13. Exiting the facility will follow the End Point Locations procedures discussed below.

\section{End Point Locations}

The final waiting location, prior to evacuation from the building, for the patient within the affected facility is called the End Point Location. As with the Hold Point Locations, there will be one for each triage level that will ideally be on the ground floor. The ideal zone for each triage level is demonstrated in Table 1.

Figure 2 shows a basic blueprint with four potential exits that will be used for a discussion with End Point Locations. The color of the area is showcasing the triage level that will be held there. In Figure 2, we are assuming there are three exits and one entrance. The entrance, labeled Influx, will be the main Emergency Department entrance and ambulance bays. This entrance is assumed to stay an entrance because if

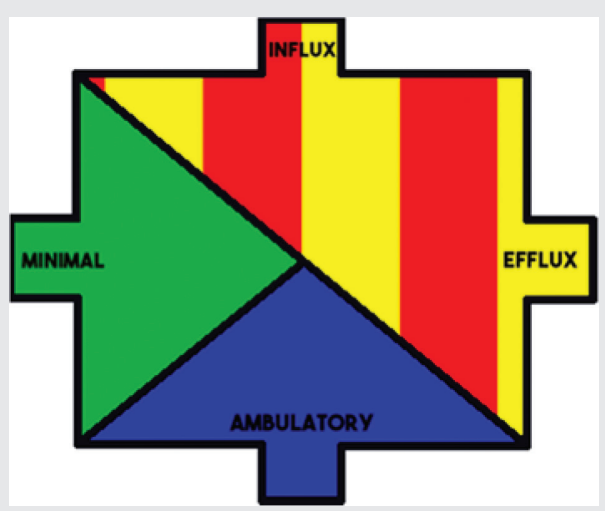

Figure 2. End Point Locations.

the hospital is experiencing an evacuation, there is a high chance that the surrounding community experienced the same devastating effects. Therefore, the Emergency Department needs to stay open to allow for potential patients to receive care. The Emergency Department must be monitored to meet the needs of the community and to remain at an optimal safety level for the current patients.

\section{Results}

A 300-bed hospital in Texas has approved an Evacuation Policy based on these standards in Texas. The plan is to implement this Evacuation Policy starting in the fall with mandatory training for all employees. Additionally, there will be a drill in the Fall of 2018 involving the NICU to test out any shortcomings with this plan.

\section{Discussion}

During a pre-approval meeting, various concerns emerged about the new Evacuation Policy. Three discussion points were mentioned: Automatically unlocking doors, the Document Pouch, and the Incident Command.

The issue of automatically locking doors would raise a concern for smooth flow of patients in and out 
of the hospital during evacuation. There would be an assigned individual from the security team in charge of unlocking the doors in the event they did not automatically unlock. Currently the nurses are still able to unlock the doors with their ID badges as needed.

There was a charge to each department within the hospital to start preparing of a Document Pouch in which there will be documents, supplies, and medications necessary for their patients for 72 hours in the event of an evacuation. In the event that they do not have the supplies or medications, they were to contact the associated departments to put requests in for the needed equipment.

The concern of who was in charge was brought up at the meeting. The staff wanted to know who was in charge of each position within the Incident Command Center. At the time, none of the positions were officially assigned, but they would be assigned to the CEO, CMO, COO, and the Safety Officer.

\section{Acknowledgements}

We would like to thank Cindi Kenady and fellow colleague Joshua Montano for the project idea and continued support over the summer. Additionally, we would like to acknowledge each of the departments for their expertise in developing this evacuation policy.

Article citation: Barry C, Ali Z. Development of an evacuation policy at a healthcare facility. The Southwest Respiratory and Critical Care Chronicles 2018;6(26):41-45

From: Texas Tech University Health Sciences Center School of Medicine in Lubbock, Texas

Submitted: 9/3/2018

Accepted: 10/10/2018

Reviewer: James Johnson Pharm D, MPA, FACHE Conflicts of interest: none This work is licensed under a Creative Commons Attribution-ShareAlike 4.0 International License

\section{REFERENCES}

1. DQE. Evacuation Program. DQE On Demand. 11 June. 2018.

2. Lucile Packard Children's Hospital. Preplanning Disaster Triage for Pediatric Hospitals. Lucile Packard Children's Hospital, 2014.

3. Mckee, Bob. Patient Evacuation During a Hurricane. Boston University Healthcare Emergency Management Program: Everbridge Webinar. 28 Sept. 2017. https://www.youtube.com/ watch?v=094GaZhvvl8 